\title{
Propagation of activity-dependent synaptic depression in simple neural networks
}

\author{
Reiko Maki Fitzsimonds, Hong-jun Song \& Mu-ming Poo \\ Department of Biology, University of California at San Diego, La Jolla, California 92093-0357, USA
}

\begin{abstract}
Triple whole-cell recordings from simple networks of cultured hippocampal neurons show that induction of long-term depression at glutamatergic synapses is accompanied by a back propagation of depression to input synapses on the dendrite of the presynaptic neuron. The depression also propagates laterally to divergent outputs of the presynaptic neuron and to convergent inputs on the postsynaptic neuron. There is no forward propagation of depression to the output of the postsynaptic neuron and no presynaptic propagation accompanying long-term depression at GABAergic synapses. Activity-induced synaptic modification is therefore not restricted to the activated synapse, but selectively propagates throughout the neural network.
\end{abstract}

The development and plasticity of the nervous system involve activity-dependent modification of synaptic connections ${ }^{1-3}$. Longterm potentiation (LTP) and long-term depression (LTD) of central synapses induced by repetitive activity in various regions of the brain have been implicated as the cellular mechanisms that underlie learning and memory ${ }^{4-7}$. These modifications are known to be input-specific, so only activated pathways are affected. But within the vicinity of activated pathways, other non-activated synapses also become modified, leading to more distributed synaptic modifications within a neural network. For example, LTP generated at one synaptic input to a CA1 hippocampal neuron was found to spread to adjacent synapses on the same or on a different postsynaptic neuron $^{8-11}$ and to result in LTD in more distant neurons ${ }^{12,13}$. Such spread of synaptic modification appears to depend on membranepermeable or secreted factors released by the activated synapse, and the extent of influence on other synapses depends on their physical proximity. On the other hand, the spread of synaptic modification can also be mediated by signalling within the neuron. On the postsynaptic side, LTP at one synaptic input can result in heterosynaptic modification of other convergent inputs ${ }^{14-19}$. On the presynaptic side, changes may spread through the cytoplasm to other divergent outputs. In Xenopus nerve-muscle cultures, LTD induced at one neuromuscular synapse can propagate to other synapses made by the same neuron onto other myocytes, apparently by signalling within the neuronal cytoplasm ${ }^{20}$. A similar presynaptic cytoplasmic mechanism may also account for the spread of shortterm depression of inhibitory synapses observed in cerebellar slices $^{21}$.

Here we find that induction of LTD at glutamatergic synapses within small networks of cultured hippocampal neurons is accompanied by a retrograde spread of depression to synapses on the dendrites of the presynaptic neurons (back propagation). The depression also spreads laterally to synapses made by divergent outputs of the presynaptic neuron (presynaptic lateral propagation) or to convergent inputs on the postsynaptic neurons (postsynaptic lateral propagation). In contrast, there was no forward propagation of depression to output synapses of the postsynaptic neuron. Moreover, we found no evidence for back propagation or presynaptic lateral propagation of depression resulting from LTD induced at GABAergic synapses. Taken together, these results indicate that there is an extensive yet selective distribution of activity-induced synaptic changes within the neural network. Furthermore, the discovery of back propagation of LTD indicates that there is an axon-dendritic flow of information which conveys signals from the outputs of a neuron to its inputs. It gives added credence to the idea that the back-propagation algorithm, which was used effectively for supervised learning in artificial neural networks ${ }^{22,23}$, may be implemented in simple biological neural networks.

\section{Triplet networks of hippocampal neurons}

Low-density cultures of hippocampal neurons were prepared from embryonic day (E) 18-20 rat embryos ${ }^{24}$ and used for experiments 10-14 days after plating. For the present study we searched for isolated groups of three interconnected hippocampal neurons (triplets) located in the microscopic field of view (a diameter of $\sim 950 \mu \mathrm{m}$ ) in the absence of other neurons (Fig. 1a). The connectivity in the triplet was examined by simultaneous whole-cell perforated patch recording from the soma of all three neurons. For each neuron, we recorded glutamatergic (excitatory) postsynaptic currents (EPSCs) or GABAergic (inhibitory) postsynaptic currents (IPSCs) in response to brief step-depolarizations initiated in each neuron under voltage-clamp conditions $\left(V_{c}=-80 \mathrm{mV}\right)$. By examining the latency of onset, the time course and the reversal potential of synaptic currents, all monosynaptic connections among three neurons in the isolated triplet and autaptic connections made by each neuron onto itself were characterized. As shown in Fig. 1b, c, the latency of onset of monosynaptic EPSCs and IPSCs following a 1-ms presynaptic depolarization had a mean value in the range of 1.5 to $2.6 \mathrm{~ms}$, whereas polysynaptic responses exhibited much longer latencies $>5 \mathrm{~ms}$ ). We have thus defined the monosynaptic connection by the presence of postsynaptic currents that exhibit a latency $\leq 2.6 \mathrm{~ms}$ and a monotonic rising phase. The monosynaptic connections used here had a mean latency of $1.8 \pm 0.4$ and $1.5 \pm 0.4 \mathrm{~ms}$ ( $n=80$ each) for EPSCs and IPSCs, respectively (see also Fig. 1d, e). For an unambiguous interpretation, experiments were done only on triplets with limited reciprocal synaptic connections. Many neurons in the triplets form autapses, which share physiological properties with synapses ${ }^{25-27}$. For simplicity of description, we used the terms 'synapse' and 'autapse' to refer to the ensemble of all synaptic and autaptic contacts (or boutons) made by a neuron.

\section{Induction of LTD}

We first characterized the induction of LTD at both glutamatergic and GABAergic synapses in these hippocampal cultures. Pairs of 
Table 1 Summary of propagation of synaptic depression in small neural networks

\begin{tabular}{|c|c|c|c|c|c|c|}
\hline \multirow[b]{2}{*}{ Network configuration } & \multicolumn{2}{|c|}{ Site of LTD induction } & \multicolumn{2}{|c|}{ Site of propagation } & \multirow[b]{2}{*}{$N \dagger$} & \multirow{2}{*}{$\begin{array}{c}\text { Propagation } \\
\text { Yes/No }\end{array}$} \\
\hline & EPSC/IPSC & Depression (\%)* & EPSC/IPSC & Depression (\%)* & & \\
\hline (1) & $\begin{array}{l}\text { (1) Back prop } \\
\text { EPSC } \\
\text { EPSC } \\
\text { IPSC } \\
\text { IPSC }\end{array}$ & $\begin{array}{l}43.5 \pm 4.9 \ddagger \\
49.8 \pm 7.0 \ddagger \\
48.3 \pm 7.6 \ddagger \\
52.2 \pm 11.5 \ddagger\end{array}$ & $\begin{array}{l}\text { EPSC } \\
\text { IPSC } \\
\text { EPSC } \\
\text { IPSC }\end{array}$ & $\begin{array}{c}27.6 \pm 3.0 \neq \\
48.4 \pm 7.5 \neq \\
4.7 \pm 2.3 \\
-6.0 \pm 10.2\end{array}$ & $\begin{array}{r}12 \\
5 \\
4 \\
5\end{array}$ & $\begin{array}{l}\text { Yes } \\
\text { Yes } \\
\text { No } \\
\text { No }\end{array}$ \\
\hline & $\begin{array}{l}\text { Forward prop } \\
\text { EPSC } \\
\text { EPSC } \\
\text { IPSC } \\
\text { IPSC }\end{array}$ & $\begin{array}{l}40.6 \pm 4.9 \ddagger \\
24.8 \pm 3.5 \ddagger \\
53.0 \pm 19.9 \ddagger \\
68.3 \pm 1.8 \ddagger\end{array}$ & $\begin{array}{l}\text { EPSC } \\
\text { IPSC } \\
\text { EPSC } \\
\text { IPSC }\end{array}$ & $\begin{array}{r}4.5 \pm 3.1 \\
-8.3 \pm 7.4 \\
3.7 \pm 3.2 \\
-1.3 \pm 4.7\end{array}$ & $\begin{array}{l}3 \\
4 \\
3 \\
3\end{array}$ & $\begin{array}{l}\text { No } \\
\text { No } \\
\text { No } \\
\text { No }\end{array}$ \\
\hline$(3)$ & $\begin{array}{l}\text { (2) Presynapt } \\
\text { EPSC } \\
\text { IPSC }\end{array}$ & $\begin{array}{l}\text { oropagation } \\
\begin{array}{r}43.8 \pm 6.3 \ddagger \\
51.2 \pm 5.3 \ddagger\end{array}\end{array}$ & $\begin{array}{l}\text { EPSC } \\
\text { IPSC }\end{array}$ & $\begin{array}{c}41.9 \pm 7.0 \ddagger \\
3.7 \pm 1.9\end{array}$ & $\begin{array}{r}10 \\
6\end{array}$ & $\begin{array}{l}\text { Yes } \\
\text { No }\end{array}$ \\
\hline & $\begin{array}{l}\text { (3) Postsynan } \\
\text { EPSC } \\
\text { EPSC } \\
\text { IPSC } \\
\text { IPSC }\end{array}$ & 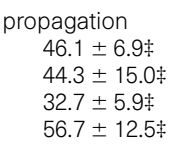 & $\begin{array}{l}\text { EPSC } \\
\text { IPSC } \\
\text { EPSC } \\
\text { IPSC }\end{array}$ & $\begin{array}{l}29.5 \pm 5.9 \ddagger \\
49.3 \pm 17.2 \ddagger \\
20.7 \pm 4.2 \ddagger \\
19.2 \pm 9.4 \ddagger\end{array}$ & $\begin{array}{l}6 \\
3 \\
3 \\
3\end{array}$ & $\begin{array}{l}\text { Yes } \\
\text { Yes } \\
\text { Yes } \\
\text { Yes }\end{array}$ \\
\hline
\end{tabular}

* The value of depression represents the percentage reduction in the amplitude of EPSCs and IPSCs during the first 10-15 min following the induction of LTD.

$\uparrow N$, total number of triplets examined for each condition.

$\ddagger$ Significantly different from zero $(P<0.01$, Mann-Whitney test).

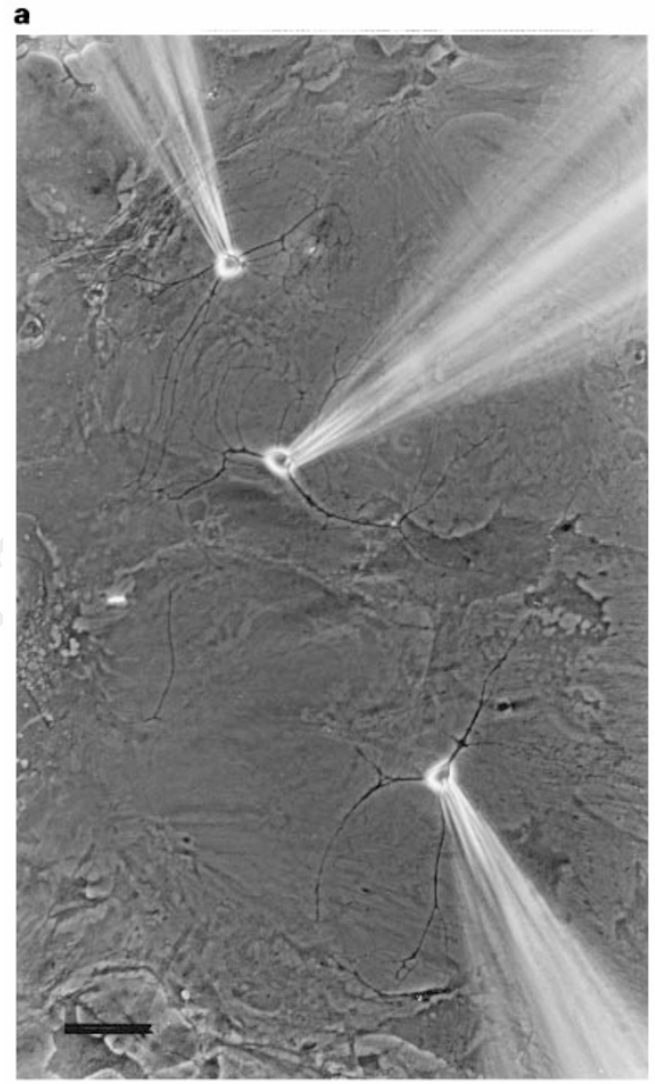

Figure 1 Simple networks of hippocampal neurons in culture. a, A phase-contrast microscopic image of a triplet network in a 10-day-old hippocampal culture, together with three pipettes used for whole-cell perforated patch recording. Scale bar, $50 \mu \mathrm{m}$. b, c, Distribution of the latency of onset of EPSCs and IPSCs recorded from four different pairs of neurons. Whole-cell recordings were made at the soma of both pre- and postsynaptic neurons in voltage-clamp mode $\left(V_{c}=-80 \mathrm{mV}\right)$. Step-depolarizations ( $+100 \mathrm{mV}, 1 \mathrm{~ms}$ ) were applied to the presynaptic neuron and postsynaptic membrane currents were monitored. Samples of successive
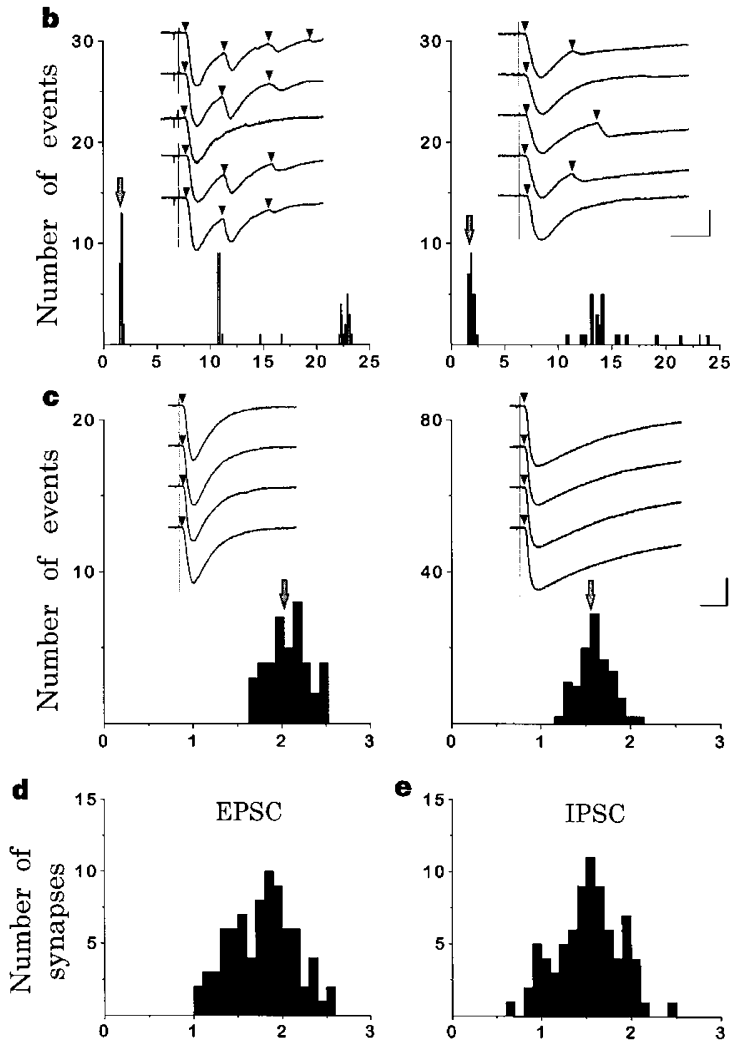

Synaptic delay (ms)

evoked synaptic currents are shown in the inset associated with each histogram. The latency was defined as the time interval between the end of 1-ms stimuli and the onset of synaptic currents. Arrowheads mark the onset time for mono- or polysynaptic responses. In c, arrows mark the mean latency for monosynaptic EPSCs (left) and IPSCs (right). Note the occasional failures in evoked polysynaptic responses in b. Scales: 20 ms, 200 pA for b; 10 ms, 200 pA for c.d, e, Distribution of the mean latency for a large number of monosynaptic glutamatergic (d) and GABAergic (e) connections ( $n=80$ each) within the triplets used here. 
hippocampal neurons were simultaneously recorded and the presynaptic neuron was stimulated repetitively under current-clamp to fire 1 -s trains of action potentials $(5 \mathrm{~Hz})$ with 1 -s intervals for $6 \mathrm{~min}$ (a total of 900 stimuli) in the presence of a sustained postsynaptic depolarization $\left(V_{c}=-50 \mathrm{mV}\right)$. This paired stimulation gave a persistent reduction in the amplitude of the EPSCs or IPSCs at glutamatergic and GABAergic synapses, respectively (Fig. 2a, b). The reduction during the first $10-15 \mathrm{~min}$ following the stimulation was $49.5 \pm 0.4 \%$ (s.e.m., $n=10$ ) and $34.6 \pm 0.5 \%$ (s.e.m., $n=6$ ) for EPSCs and IPSCs, respectively, and it persisted for at least $20 \mathrm{~min}$ (Fig. 2c, d). Concurrent postsynaptic depolarization was necessary for the induction of LTD by the present stimulation protocol; when the postsynaptic neurons was voltage-clamped at $-80 \mathrm{mV}$ during the period of repetitive presynaptic stimulation, no LTD was observed (Fig. 2c, d). Furthermore, the induction of LTD at glutamatergic synapses was largely abolished by the presence of (士)-2-amino-5-phosphonopentanoic acid (AP5, $50 \mu \mathrm{M}$; Fig. 2e), an antagonist that blocks the NMDA ( $N$-methyl-D-aspartate) subtype of glutamate receptors, whereas LTD at GABAergic synapses was unaffected by the same AP5 treatment (Fig. 2f). These properties of the induction of LTD agree with those previously described for glutamatergic synapses in hippocampal cultures ${ }^{26}$ and slices $^{28-32}$. The properties of GABAergic LTD remain to be further characterized.

\section{Back propagation of synaptic depression}

Serially connected triplets were first used for studying the propagation of LTD. Figure 3a shows an example consisting of only glutamatergic synapses. Matrix tabulation of representative averages of synaptic and autaptic currents is used to depict the connectivity and time-dependent synaptic changes. Alternating test stimuli were applied to neurons 1 and 2 at low frequency $(0.06 \mathrm{~Hz})$ to monitor the synaptic efficacy of connections, except during the 6min period when LTD was induced at the glutamatergic synapse (E) from neuron 2 to $3\left(\mathrm{E}_{2-3}\right)$. During the induction period, neuron 2 was stimulated repetitively to fire action potentials (see above), while neuron 3 was voltage-clamped at $-50 \mathrm{mV}$. Neuron 1 remained voltage-clamped at $-80 \mathrm{mV}$ throughout the experiment. Immediately after the stimulation, the amplitude of EPSCs at $\mathrm{E}_{2-3}$ was persistently reduced. EPSCs at $\mathrm{E}_{1-2}$ were also reduced, indicating a back propagation of depression from the axonal output of neuron 2 to its dendritic input. In a different triplet consisting of both glutamatergic and GABAergic neurons (Fig. 3b), induction of LTD at $\mathrm{E}_{2-3}$ was also accompanied by a depression of the GABAergic

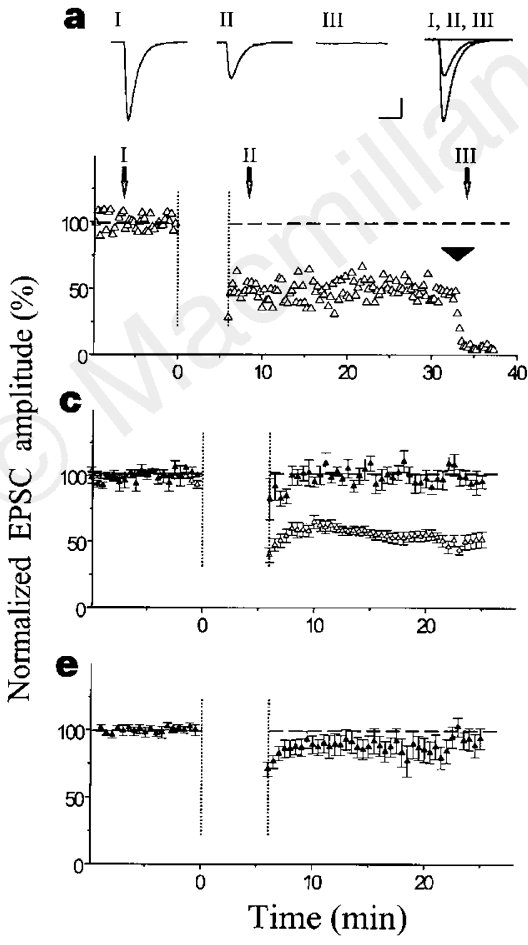

Figure 2 Induction of LTD at glutamatergic and GABAergic synapses in hippocampal cultures. a, b. Examples of recording at glutamatergic (a) and GABAergic (b) synapses. Data points depict the amplitudes of EPSCs or IPSCs before and after the induction of LTD, normalized to the mean value recorded (as $100 \%$ ) before the induction. For the induction of LTD, the presynaptic neuron was stimulated repetitively under current clamp to fire 1-s trains of action potentials ( $5 \mathrm{~Hz}$ ) with 1-s intervals for 6 min (a total of 900 stimuli) in the presence of a sustained postsynaptic depolarization $\left(V_{c}=-50 \mathrm{mV}\right)$. Dotted lines delineate the period of repetitive stimulation. Arrowheads indicate the time of application of glutamate antagonists ( $50 \mu \mathrm{M}$ AP5; $10 \mu \mathrm{M} C N Q X)$ and $\mathrm{GABA}_{\mathrm{A}}$ receptor antagonists (10 $\mu \mathrm{M}$ bicuculline) in $\mathbf{a}$ and $\mathbf{b}$, respectively. Traces of EPSCs and IPSCs (average of 5 consecutive events) before (I) and after (II) the induction of LTD and after addition of receptor antagonists (III) are shown above. Note the slower decay of the IPSCs. Scales: 20 ms, 200 pA for a; 40 ms, 200 pA for b. c, d, Summary of

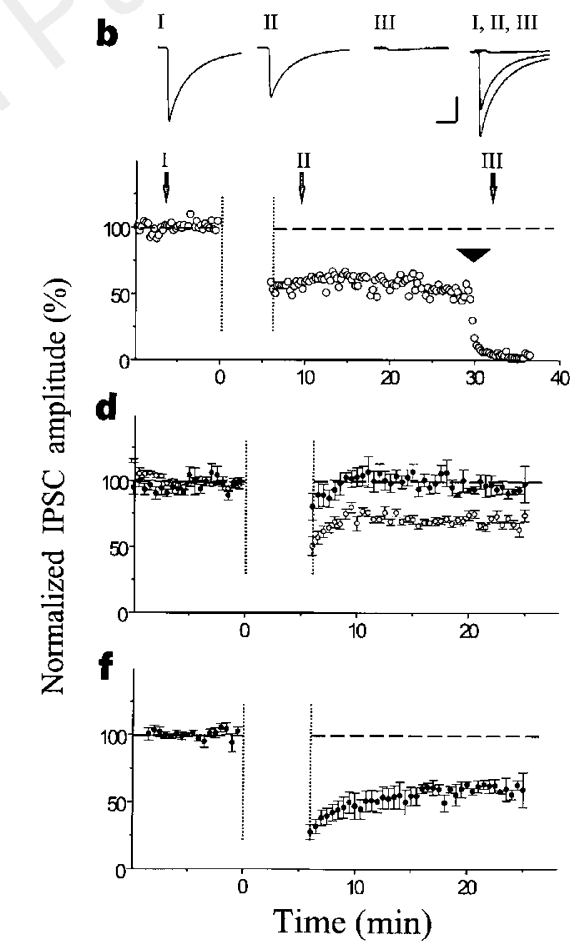

experiments on the induction of LTD at glutamatergic (c) and GABAergic synapses (d). When the postsynaptic cell was voltage-clamped at $-50 \mathrm{mV}$ during the repetitive presynaptic stimulation, there was significant and persistent reduction of EPSCs (c, open triangles; $n=10$ ) and IPSCs (d, open circles; $n=6$ ) $(P<0.05$, Student's paired $t$-test). In contrast, when the postsynaptic neuron was voltage-clamped at $-80 \mathrm{mV}$ during stimulation, no significant change in EPSCs (c, filled triangles; $n=5$ ) or IPSCs (d, filled circles; $n=5)$ was observed $(P>0.05$, Student's paired $t$-test). Data points represent mean \pm s.e.m. e, $\mathbf{f}$, Effect of AP5 on the induction of LTD. The same as that described in $\mathbf{c}$ and $\mathbf{d}$, except that AP5 $(50 \mu \mathrm{M})$ was added in the extracellular bath solution throughout the experiment. The degree of depression was greatly reduced for glutamatergic $\operatorname{LTD}(\mathbf{e} ; n=6)$, whereas GABAergic LTD was unaffected $(\mathbf{f} ; n=4)$. Both the residual depression at glutamatergic synapses and the depression at GABAergic synapses were significant $(P<0.05$, Student's paired $t$-test). 
synapse (I) from neuron 1 to $2\left(\mathrm{I}_{1-2}\right)$. Note that there was no depression of the autapse made by neuron $1\left(\mathrm{E}_{1-1}\right.$ or $\mathrm{I}_{1-1}$; Fig. 1a, b), but the reciprocal synapse $\mathrm{E}_{2-1}$ was depressed in both cases. The depression of $E_{2-1}$ suggests a lateral propagation of LTD to divergent axonal outputs of the presynaptic neuron (see below).

The results from all serial triplets recorded are summarized in Fig. 3c, $\mathrm{d}$ and Table 1. In control experiments (Fig. 3c), we found no change in the synaptic efficacy of either $\mathrm{E}_{1-2}$ or $\mathrm{I}_{1-2}$ after applying repetitive stimulation to neuron 2 while voltage-clamping neuron 3 at $-80 \mathrm{mV}$. Thus coincident pre- and postsynaptic excitation is required not only for the induction of LTD, but also for the back propagation of depression. The same stimuli resulted in a persistent depression of both synapses $\mathrm{E}_{2-3}$ and $\mathrm{E}_{1-2}\left(\right.$ or $\mathrm{I}_{1-2}$ ) when neuron 3 was concurrently depolarized to $-50 \mathrm{mV}$ (Fig. 3d). The average steady depression at back-propagation site $\mathrm{E}_{1-2}$ or $\mathrm{I}_{1-2}$ was significantly lower than that of the LTD induced at $\mathrm{E}_{2-3}(P<0.02$, Student's $t$-test; Fig. $3 \mathrm{~d}$ and Table 1). In these experiments, we noted in many cases an initial short-term depression following the termination of the repetitive stimuli at the site of LTD induction but not at the propagation site (Fig. 3b), consistent with the absence of repetitive presynaptic stimulation at the propagation site.

In contrast to the back propagation of depression associated with glutamatergic LTD, we found no evidence for back propagation of depression following induction of LTD at GABAergic synapses. In the example shown in Fig. 4a, when LTD was induced at the GABAergic synapse $\mathrm{I}_{2-3}$ by the same stimulation protocol, no depression of $\mathrm{E}_{1-2}$ was observed. Back propagation was also absent in serial triplets that consisted only of GABAergic synapses. Neither was $\mathrm{I}_{2-1}$ affected by the induction of LTD at $\mathrm{I}_{2-3}$, suggesting the absence of presynaptic lateral spread of depression in GABAergic neurons (see below). The results on the back propagation of GABAergic LTD are summarized in Fig. 4b and Table 1.

\section{Forward propagation}

Serial triplets of hippocampal neurons were also used to determine
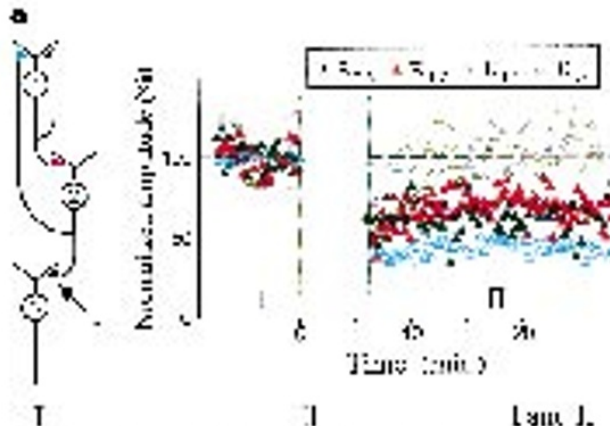

$\mathrm{T}$
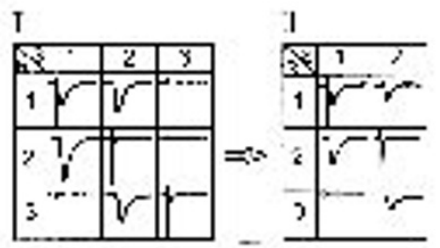

โ צแI ].
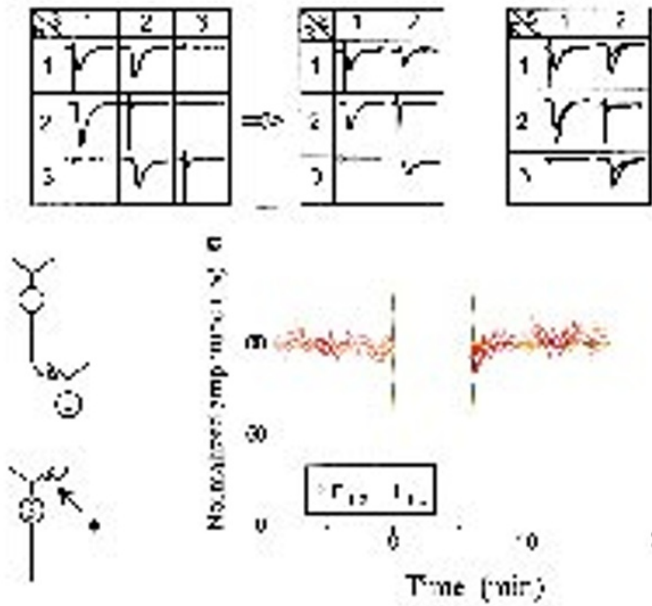

\%:

Figure 3 Back propagation of synaptic depression following the induction of LTD at glutamatergic synapses. a, An example of a glutamatergic triplet. The diagram on the left indicates the connectivity deduced from measurements of monosynaptic and autaptic currents recorded from all three neurons. The matrices below depict samples (average of 4 consecutive events) of synaptic and autaptic currents before (I) and after (II) the induction of LTD as well as superimposed currents (I and II) for comparison. S, stimulated neuron; R, recorded neuron. Note that a large stimulation artefact precedes the autaptic current. Scales: $20 \mathrm{~ms}$, $250 \mathrm{pA}$. Data points depict the amplitude of EPSCs at the autapse of neuron $1\left(E_{1-1}\right.$, white symbols) and at synapses from neuron 1 to $2\left(E_{1-2}\right.$, red), from 2 to 3 ( $E_{2-3}$, black) and from 2 to 1 ( $E_{2-1}$, blue). Amplitudes were normalized as for Fig. 2 . The same stimulation procedure as for Fig. 2 was used to induce LTD at $E_{2-3}$ (black arrow and asterisk), while neuron 1 was voltage-clamped at $-80 \mathrm{mV}$. b. An example of a triplet formed by glutamatergic and GABAergic neurons. The same as in a, except that neuron 1 was GABAergic. Note the slow time course for IPSCs elicited by neuron 1 ( $S 1$; scales: $40 \mathrm{~ms}, 200 \mathrm{pA}$ ) compared to that of EPSCs elicited by neuron 2 (S2; scales: $20 \mathrm{~ms}, 200 \mathrm{pA}$ for S2 and S3). c. Summary of control experiments. The same repetitive stimulation as that used in the induction of LTD (a) was applied to neuron 2 in serial triplets while neuron 3 was voltage-clamped at $-80 \mathrm{mV}$. Left, generic configuration for serial triplets studied. Data (mean \pm s.e.m.) represent synaptic responses at $E_{1-2}$ and $I_{1-2}(n=7$, including 3 $E_{1-2}$ and $\left.4 I_{1-2}\right)$. d. Summary of back propagation of depression following the induction of LTD at glutamatergic synapses in serial triplets. The same repetitive stimulation as that used in a was applied to neuron 2 while neuron 3 was depolarized to $-50 \mathrm{mV}$. Data points are mean \pm s.e.m. $(n=8)$. Filled triangles, EPSCs at $E_{2-3}$; red diamonds, averages of EPSCs and IPSCs at $E_{1-2}(n=6)$ and $\mathrm{I}_{1-2}$ $(n=2)$, respectively. 
Figure 4 Absence of back propagation of depression following the induction of LTD at GABAergic synapses. a, An example of a triplet consisting of both glutamatergic and GABAergic neurons. LTD was induced at the GABAergic synapse $\mathrm{I}_{2-3}$ (black) by using the same protocol as for Fig. 3, and the synaptic efficacy at $\mathrm{E}_{1-2}$ (red) and $\mathrm{I}_{2-1}$ (blue) were monitored before and after induction. Scales: $40 \mathrm{~ms}, 250 \mathrm{pA}$ for S2; $20 \mathrm{~ms}, 250 \mathrm{pA}$ for S1 and S3. b. Summary of back propagation of depression following the induction of LTD at GABAergic synapses $\left(\mathrm{I}_{2-3}\right)$ in serial triplets similar to that in a. Data represent mean \pm s.e.m. $(n=7)$. Black circles, IPSCs at $\mathrm{I}_{2-3}$; red diamonds, average synaptic currents at $\mathrm{E}_{1-2}(n=4)$ and $\mathrm{I}_{1-2}(n=3)$.
Figure 5 Absence of forward propagation of depression. a, An example of a serially connected triplet. Using the same stimulation procedure as that described in Fig. 3, LTD was induced at $E_{1-2}$. The data shown are normalized amplitudes of synaptic currents at $E_{1-2}$ (black), $E_{2-3}$ (red), and $E_{2-1}$ (blue). Scale: $20 \mathrm{~ms} ; 500 \mathrm{pA}$ for R1, $250 \mathrm{pA}$ for R2, $1 \mathrm{nA}$ for R3. b. An example of a triplet with 'circular' connection. Alternating assays of EPSCs at $E_{3-1}$ (red) and $E_{1-2}$ (blue) showed that, following the induction LTD at $E_{2-3}$ (black), depression was observed at $E_{1-2}$, but not at $E_{3-1}$. Scale: $20 \mathrm{~ms} ; 500 \mathrm{pA}$ for R1, $125 \mathrm{pA}$ for R2 and R3. c, d, Summary of all experiments on forward propagation. Schematic diagram on the left depicts the generic serial connectivity used in these studies. Induction of LTD was achieved at $E_{1-2}(\mathbf{c})$ or $\mathrm{I}_{1-2}(\mathbf{d})$, respectively, and the efficacy of either $E_{2-3}$ or $I_{2-3}$ was examined (red diamonds). Data represent mean \pm s.e.m. $(n=6$ each in $\mathbf{c}$ and $\mathbf{d}$, including $3 \mathrm{E}_{2-3}$ and $3 \mathrm{I}_{2-3}$ ).
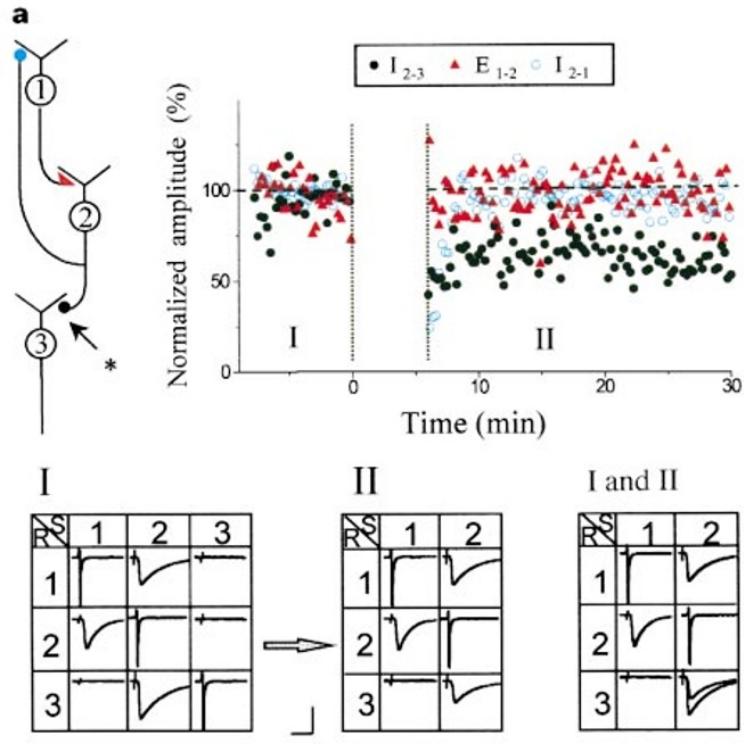

I and II
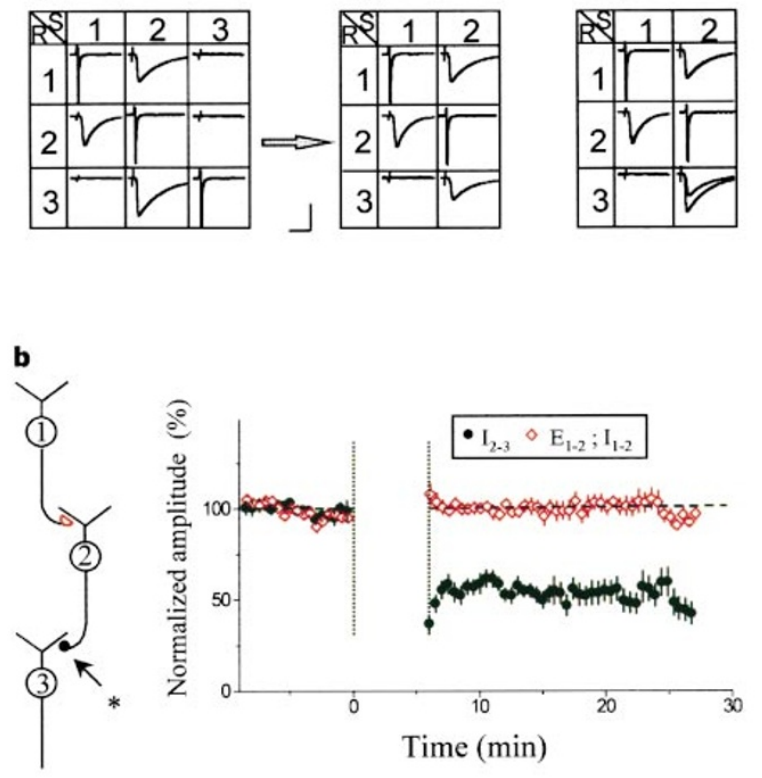
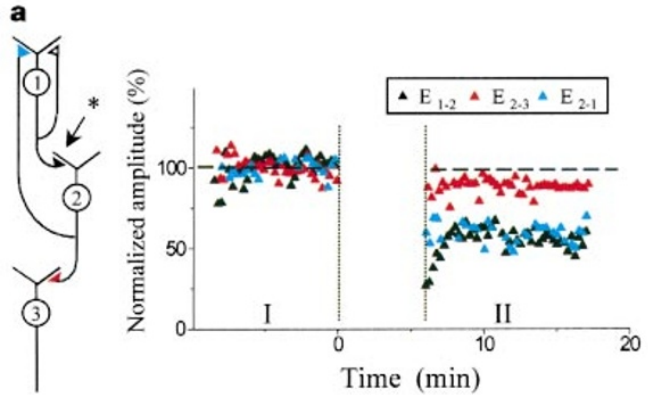

I

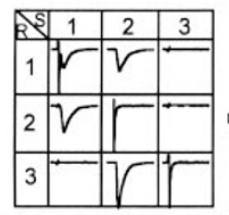

II
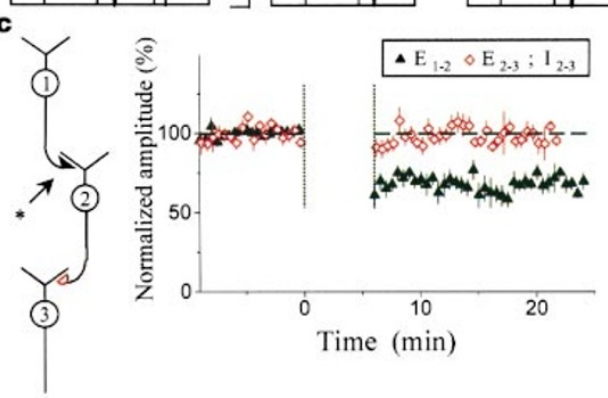

I and II

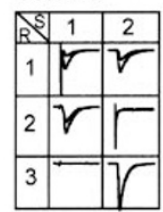

$\Rightarrow 2$

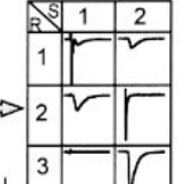

b
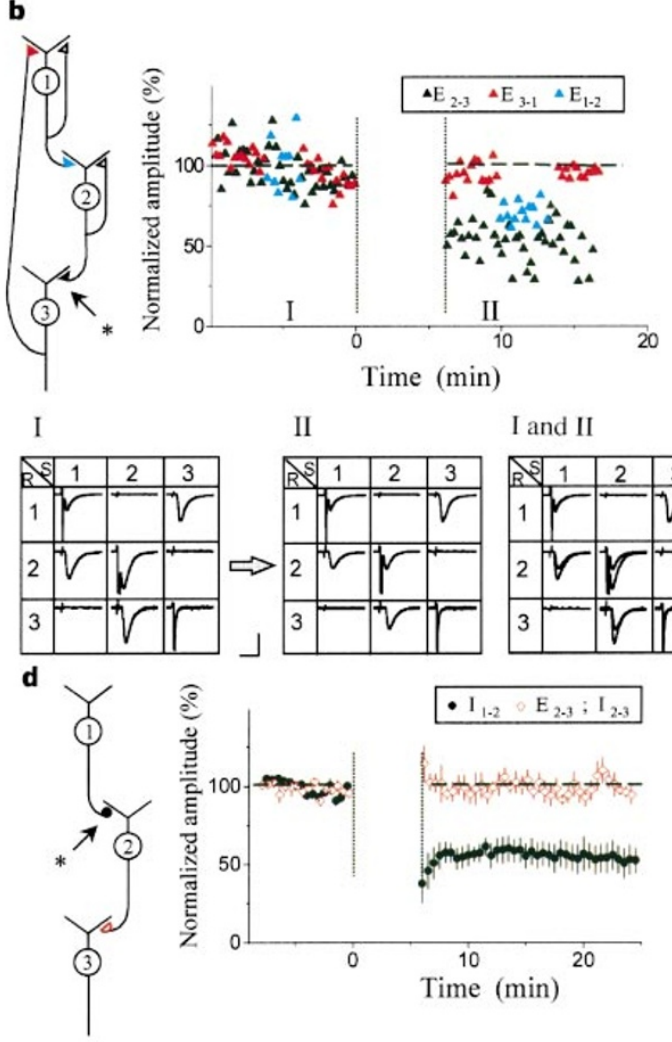

II

I and II

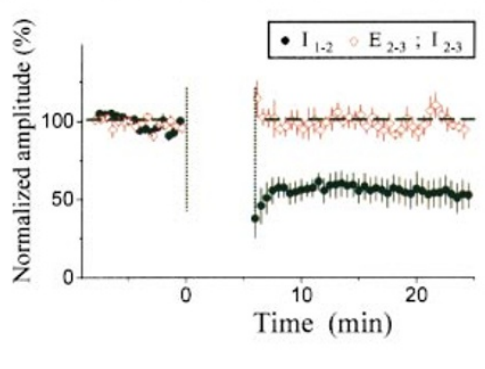



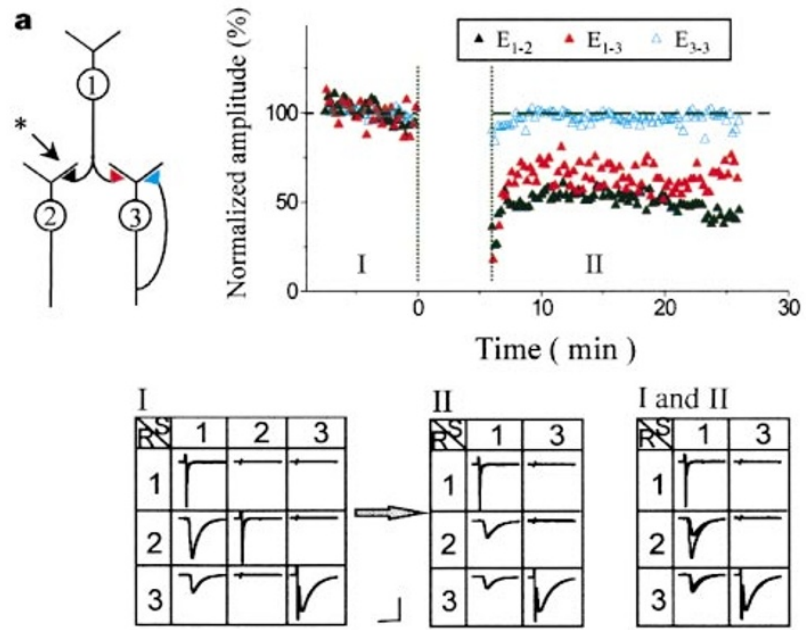

I and II
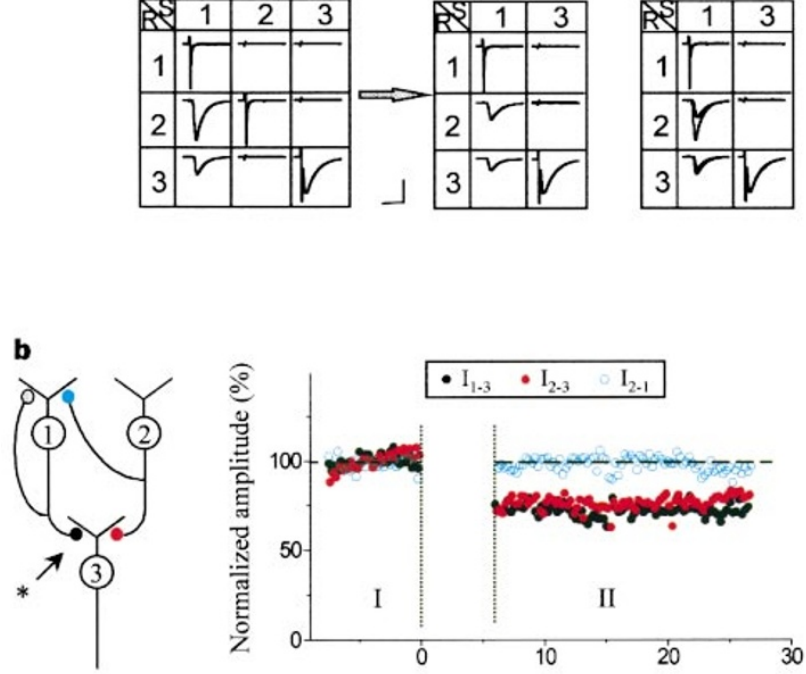

Time ( $\min )$
Figure 6 Lateral propagation of LTD. a, An example of a triplet with divergent glutamatergic outputs. For induction of LTD at $E_{1-2}$, neurons 1 and 2 were coactivated as for Fig. 3 while neuron 3 was voltage-clamped at $-80 \mathrm{mV}$. Data shown are normalized EPSCs recorded at $\mathrm{E}_{1-2}$ (black), $\mathrm{E}_{1-3}$ (red), and $\mathrm{E}_{3-3}$ (blue). Scales: $20 \mathrm{~ms} ; 1 \mathrm{nA}$ for R1, $500 \mathrm{pA}$ for R2 and R3. b. An example of a triplet with converging GABAergic inputs. Induction of LTD was achieved at $\mathrm{I}_{1-3}$ while neuron 2 was voltage-clamped at $-80 \mathrm{mV}$. Data depict normalized amplitudes of EPSCs at $\mathrm{I}_{1-3}$ (black), $I_{2-3}$ (red) and $I_{2-1}$ (blue), before and after induction of LTD at $I_{1-3}$. Scales: $20 \mathrm{~ms} ; 400 \mathrm{pA}$ for R1 and R2, $100 \mathrm{pA}$ for R3. c-h, Summary of results from experiments on lateral propagation of depression. Diagrams on the left depict the generic triplet configuration used. c, Control experiments. Repetitive stimulation of the presynaptic glutamatergic neuron (as in $\mathbf{a}$ ) in the absence of postsynaptic depolarization of neuron 2 and $3\left(V_{c}=-80 \mathrm{mV}\right)$ did not induce synaptic depression at $E_{1-3}$. Data represent mean \pm s.e.m. $(n=6)$. d, Summary of presynaptic lateral propagation of depression following induction of glutamatergic LTD. Data shown are normalized amplitudes of EPSCs (mean \pm s.e.m.; $n=6$ ) at the site of LTD induction (black) and at the other divergent output of the same presynaptic neuron (red). e, Summary of presynaptic lateral propagation of depression following the induction of GABAergic LTD. Shown are data of normalized amplitudes of IPSCs at the site of LTD induction (black) and at the other divergent output (red) $(n=4)$. f, Control experiments. Sustained depolarization of the postsynaptic cell $\left(V_{c}=-50 \mathrm{mV}\right)$ for $6 \mathrm{~min}$ in the absence of repetitive presynaptic stimulation did not induce any change in EPSCs (red, $n=4$ ) or IPSCs (black, $n=7$ ). Data represent mean \pm s.e.m. $\mathbf{g}$, Summary of postsynaptic lateral propagation of depression following the induction of glutamatergic LTD. Data depict normalized amplitudes of EPSCs at the site of LTD induction (black) and of EPSCs $(n=5)$ or IPSCs $(n=1)$ at the other convergent input on the same postsynaptic neuron (red). Data represent mean \pm s.e.m. $(n=6)$. $\mathbf{h}$, Summary of postsynaptic lateral propagation following the induction of GABAergic LTD. Data depict normalized amplitudes of IPSCs at the site of LTD induction (black) and of EPSCs $(n=3)$ or IPSCs $(n=1)$ at the other convergent input on the same postsynaptic neuron (red). Data represent mean \pm s.e.m $(n=4)$.

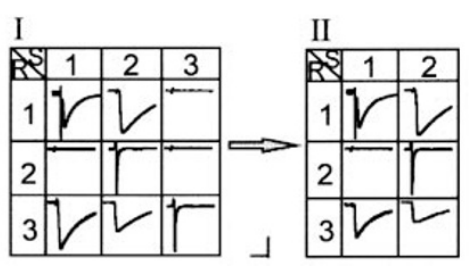

I and II

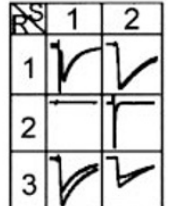

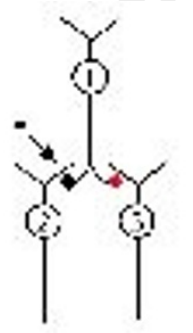

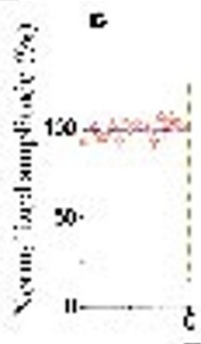<smiles>Ic1cncnc1I</smiles>

Tiure !mir.!
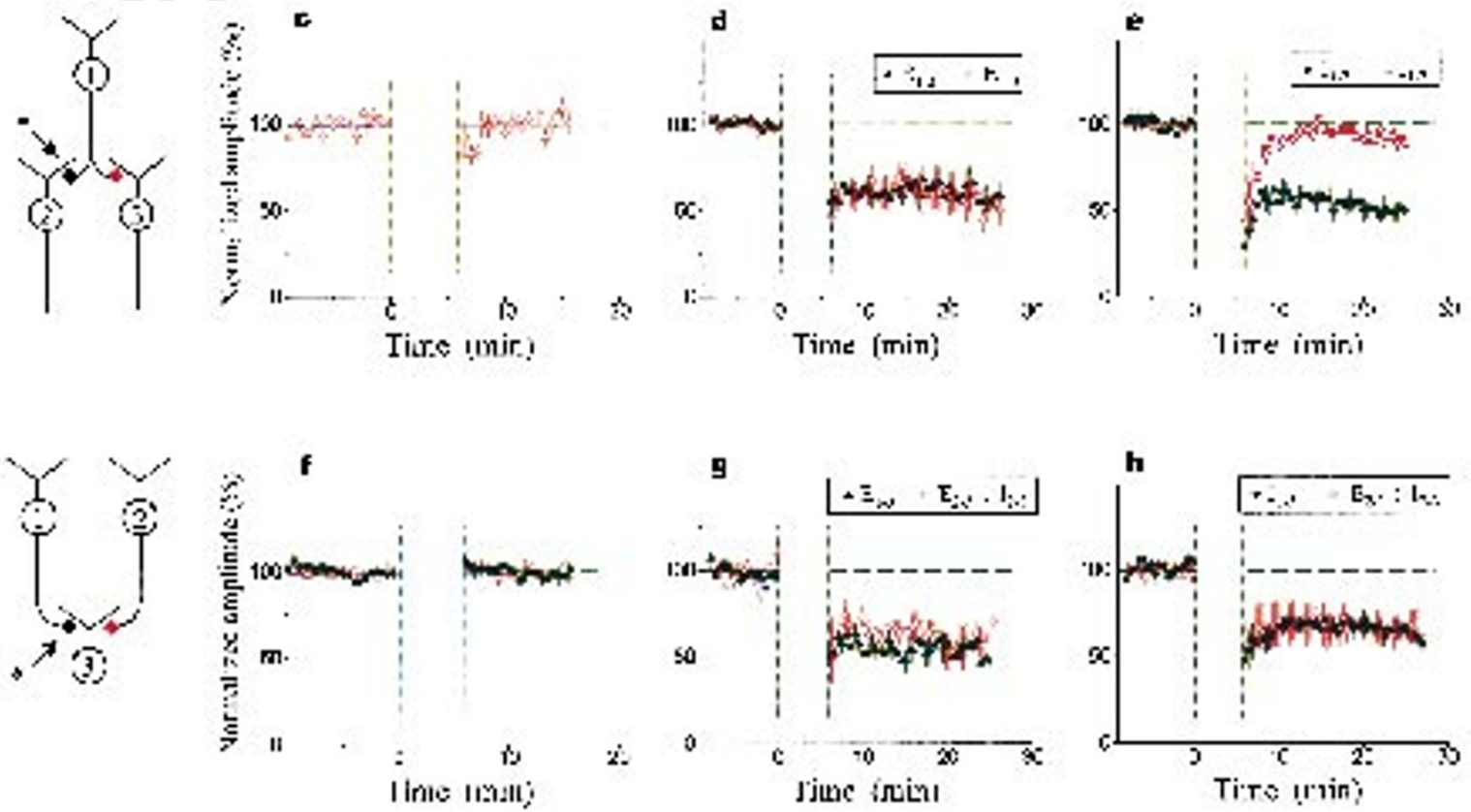
whether LTD can spread in the anterograde direction from the dendrites of the postsynaptic neuron to synapses made by its axonal terminals. In the example shown in Fig. 5a, we found that induction of LTD at $\mathrm{E}_{1-2}$ did not result in significant depression of $\mathrm{E}_{2-3}$, although the depression had back propagated to $\mathrm{E}_{2-1}$ and laterally propagated to $\mathrm{E}_{1-1}$ (see matrix in Fig. 5a). In an example of a 'circular' triplet (Fig. 5b), induction of LTD at $\mathrm{E}_{2-3}$ did not result in any depression of $E_{3-1}$, but caused significant depression of $E_{1-2}$. Thus back propagation occurred in the absence of forward propagation within the same triplet. In other experiments on serial triplets containing GABAergic neurons, we also found no evidence of forward propagation of depression to either $\mathrm{E}_{2-3}$ or $\mathrm{I}_{2-}$ 3 following the induction of LTD at $\mathrm{I}_{1-2}$. These results on the absence of forward propagation are summarized in Fig. $5 c$, $d$ and Table 1.

\section{Lateral propagation}

In addition to the serial configuration, networks of triplets with other configurations were also studied. In triplets consisting of one neuron making connections onto two other neurons (Table 1), we examined whether LTD can spread laterally between divergent outputs of the presynaptic neuron. An example of pure glutamatergic triplet is shown in Fig. 6a. LTD was induced at $\mathrm{E}_{1-2}$ by repetitive firing of neuron 1 concurrently with sustained depolarization of neuron $2\left(V_{c}=-50 \mathrm{mV}\right)$, while neuron 3 remained voltage-clamped at $-80 \mathrm{mV}$. Following the induction of LTD at $\mathrm{E}_{1-2}$, significant and persistent depression was also observed at $\mathrm{E}_{1-3}$, while $\mathrm{E}_{3-3}$ was not affected. Control experiments showed that repetitive firing of the presynaptic neuron alone, with the postsynaptic neuron 2 and 3 voltage-clamped at $-80 \mathrm{mV}$, resulted in no persistent change in the amplitude of EPSCs at either $\mathrm{E}_{1-2}$ or $\mathrm{E}_{1-3}$ (Fig. 6c). For all divergent triplets examined, we found consistent propagation of LTD to other synaptic outputs of the glutamatergic neuron and the degree of depression at the propagation site correlated with that found at the induction site (Figs $6 \mathrm{~d}, 7 \mathrm{e}$ and Table 1). Triplet networks consisting of GABAergic neurons with divergent outputs were also examined. As summarized in Fig. 6e, the same pairedstimulation of neuron 1 and 2 as described above resulted in LTD of the GABAergic synapse $\mathrm{I}_{1-2}$. However, only an initial transient depression of $\mathrm{I}_{1-3}$ was observed following the induction of LTD at $\mathrm{I}_{1-2}$, indicating an absence of lateral propagation of LTD to divergent GABAergic outputs. The lack of presynaptic lateral spread was also observed for the triplet shown in Fig. 4a. Note that the appearance of transient depression at all divergent outputs of the presynaptic neuron is consistent with short-term depression following repetitive stimulation. Overall, although the extent of LTD at GABAergic synapses was similar to that found at glutamatergic synapses, there was no spread of depression to divergent outputs of the presynaptic GABAergic neurons (Table 1).

We have also examined triplets that contained two neurons making convergent inputs on a third neuron (Table 1). An example of a triplet with two convergent GABAergic inputs is shown in Fig. 6 b. Induction of LTD at $\mathrm{I}_{1-3}$ was achieved by repetitive firing of neuron 1 in the presence of sustained depolarization of neuron 3 at $-50 \mathrm{mV}$, while neuron 2 was voltage-clamped at $-80 \mathrm{mV}$. This also resulted in a persistent depression of $\mathrm{I}_{2-3}$, indicating lateral propagation of LTD to convergent inputs on neuron 3 . However, the autapse $I_{1-1}$ and synapse $I_{2-1}$ were not affected by the induction of LTD at $\mathrm{I}_{1-3}$, consistent with the lack of presynaptic lateral and back propagation in the GABAergic neurons. Sustained postsynaptic depolarization of neuron 3 during the induction of LTD was not sufficient to cause the depression at $\mathrm{I}_{2-3}$, because depolarization of postsynaptic cells at $-50 \mathrm{mV}$ for $6 \mathrm{~min}$ by itself had no effect on either glutamatergic or GABAergic synapses (Fig. 6f). Figure $6 \mathrm{~g}, \mathrm{~h}$ summarizes results of all experiments on postsynaptic lateral propagation following the induction of LTD at glutamatergic and GABAergic synapses, respectively. Depression was similar for lateral propagation to either glutamatergic or GABAergic synapse (Table 1).

\section{Range of propagation}

Our simultaneous recordings were limited to only three neurons; thus the propagation of depression was not monitored beyond the triplet network. We noted, however, that the autapse $\mathrm{E}_{1-1}$ in triplets shown in Figs $3 a, b$ and $5 b$ was not depressed, whereas $E_{1-2}$ were depressed by back propagation in presynaptic neuron 2. Assuming that autapses may be considered as converging inputs or divergent output within the network, then the absence of depression at $E_{1-1}$ indicates that propagated depression at $\mathrm{E}_{1-2}$ does not further laterally or back propagate to a more 'upstream' neuron 1 . In the divergent triplet shown in Fig. $6 a$, the autapse $\mathrm{E}_{3-3}$ did not become depressed while depression had propagated to $\mathrm{E}_{1-3}$. Further lateral propagation was lacking in all cases with similar configuration $(n=5)$. The propagated depression at the divergent output thus appears to be incapable of further propagation to more 'downstream' synapses made by or on the postsynaptic neurons associated with that divergent output. In addition, we found that in all cases examined for postsynaptic lateral propagation $(n=7)$, autapses in neurons not directly associated with the induction of LTD were not depressed, regardless of whether LTD was induced at glutamatergic or GABAergic synapses. The lack of further propagation beyond the immediate pre- and postsynaptic neurons involved in the induction of LTD may be due to the limited degree of propagated depression. Alternatively, there may be a qualitative difference in the mechanism underlying the depression at the induction and propagation sites.

\section{Potential cellular mechanisms}

We have observed extensive propagation of depression from the site of induction of LTD to other synaptic sites within a triplet network. The propagation may result from the spread of 'depressive' signals either within the pre- or postsynaptic neurons or through the extracellular space. In these cultures, multiple synaptic (and autaptic) contacts made on each neuron were usually distributed rather haphazardly. The average spatial distributions of synaptic and autaptic sites with respect to sites of LTD induction are likely to be similar, regardless of their functional position within the serial network. However, the depression did not spread non-selectively to all synapses and autapses within the network. This was shown by the absence of forward propagation, whereas lateral and back propagation were observed within the same triplet (Fig. 5) and by the lack of effect on autapses formed on the neuron exhibiting propagated depression in the example shown in Figs 3, 5b, 6a. These findings, together with the fact that constant perfusion of fresh medium was applied to the culture throughout the recording period, are evidence against extracellular diffusible factors being mediators of the spread of depression.

Previous studies of LTD at developing neuromuscular junctions $s^{33,34}$ and in brain slices ${ }^{29,30,35}$ have shown that, although postsynaptic influx of $\mathrm{Ca}^{2+}$ is required for its induction, LTD is expressed as a presynaptic reduction of evoked transmitter release. This suggests the existence of retrograde signalling from the post- to the presynaptic neuron. The retrograde signal may generate a secondary cytoplasmic factor in the presynaptic glutamatergic neuron for the back and lateral propagation of depression. The absence of back and lateral propagation of GABAergic LTD suggests a postsynaptic expression of LTD at these synapses, without involvement of retrograde presynaptic modification. As there is no forward propagation of depression for both glutamatergic and GABAergic synapses, the depressive signal produced at the site of LTD induction appears to be incapable of propagation in the anterograde direction. The postsynaptic site may fail to generate a necessary secondary signal, the signal may not be transported in the anterograde direction, or the output terminal of the postsynaptic cell may not be susceptible to modification by the signal. 
Finally, although cytoplasmic signalling would explain a selective propagation of depression, we cannot exclude the possibility that extracellular depressive signals are involved. Repetitive paired stimulation during the induction of LTD may have rendered all synapses associated with the pre- or postsynaptic neuron selectively susceptible to an extracellular depressive signal.

A clue to the mechanisms underlying back propagation of depression may be provided by the extent of back-propagated depression in different triplets. As shown in Fig. 7a, the degree of depression observed at either $\mathrm{E}_{1-2}$ or $\mathrm{I}_{1-2}$ after the induction of LTD at $\mathrm{E}_{2-3}$ was strongly correlated with the initial amplitude of EPSCs or IPSCs at $\mathrm{E}_{1-2}$ or $\mathrm{I}_{1-2}$, respectively. A weak correlation was found between the degree of the propagated depression and the rise time of the synaptic currents (Fig. 7b). In contrast, for either the induced LTD or forward-propagated depression, there was no correlation between the degree of depression and the initial amplitude of the synaptic current (Fig. 7c). The amplitude and rise time may reflect the average electrotonic distance of various synaptic sites from the soma; those on more distal dendrites elicit currents of smaller amplitude and longer rise time. The correlation found for the back-propagated depression is thus consistent with the notion of cytoplasmic retrograde signals, which decrement with the cytoplasmic distance between the axonal endings and synaptic sites on the dendrite. On the other hand, the amplitude of the synaptic current can also be attributed to other intrinsic properties of the synapse, for example the number of synaptic boutons, the effectiveness of the synaptic contact, or the probability of transmitter secretion. These properties may result in a differential susceptibility of a synapse to the retrograde signal, resulting in the observed correlation between the degree of depression at the back-propagation site and the initial amplitude of the postsynaptic currents at that site. Indeed, synapses with different release probability show different degrees of short-term plasticity ${ }^{36}$.

The presence of reciprocal connections between neurons 1 and 2 $\left(E_{1-2}\right.$ and $\left.E_{2-1}\right)$ was neither required nor related to the degree of propagated depression, as shown by the similar range of depression for triplets without $\mathrm{E}_{2-1}$ (Figs $7 \mathrm{a}, \mathrm{b}, 5 \mathrm{~b}$ ). However, we cannot exclude the potential contribution of recurrent presynaptic inhibition or facilitation, which escapes detection by our recording methods. The mean amplitude of synaptic currents for synapses used for examining forward propagation covered a range of values similar to that used for studying back propagation (Fig. $7 \mathrm{c}$ ), indicating that the lack of forward propagation was not due to a biased sampling of forward synapses with smaller synaptic currents. We found no correlation between the degree of back-propagated depression and induced depression (Fig. 7d), suggesting that the extent of induced LTD bears no relation to the retrograde influence received by the dendrite of the presynaptic neuron. In contrast, the extent of depression due to presynaptic lateral propagation is linearly correlated with that of the induced LTD (Fig. 7e). There was no obvious correlation between the postsynaptic lateral depression and the induced depression (Fig. 7f). These differences indicate
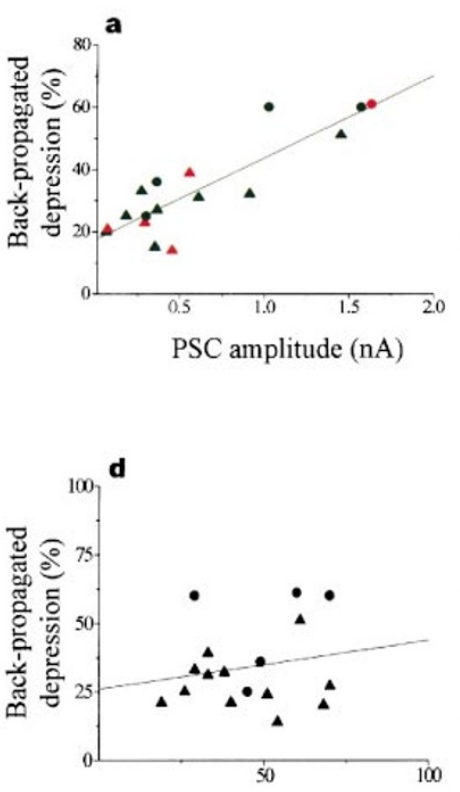

Induced depression (\%)

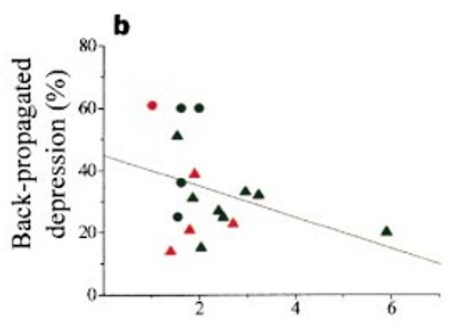

PSC risetime (ms)

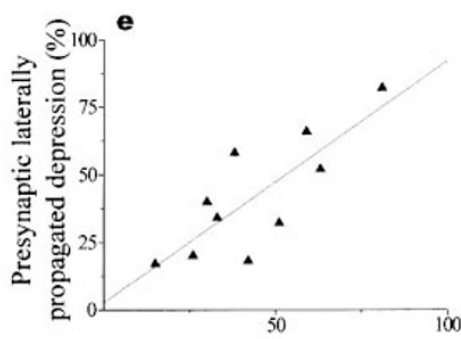

Induced depression (\%)
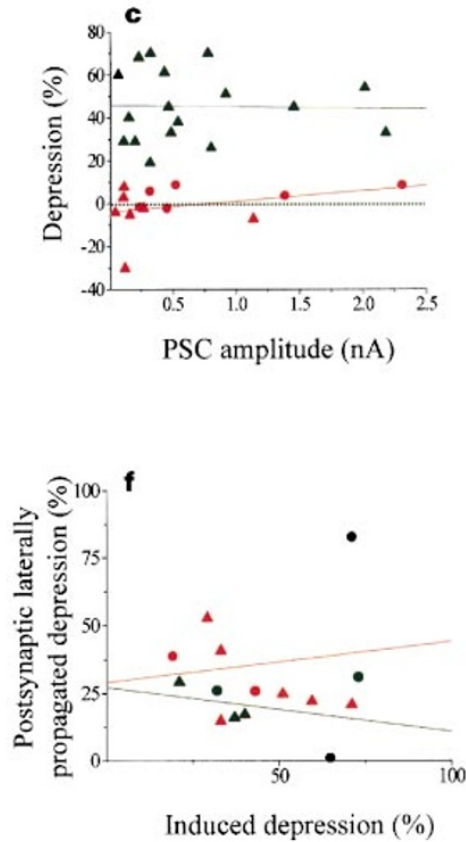

Figure $\mathbf{7} \mathbf{a}, \mathbf{b}$, Correlation between the degree of back propagation of synaptic depression and synaptic properties. The percentage of reduction in the mean amplitude of EPSCs (triangles, $n=12$ ) or IPSCs (circles, $n=5$ ) at the backpropagation site of presynaptic neurons during the first 10-15 min following the induction of LTD is plotted against the initial mean amplitude (a) or the risetime (b) of the synaptic currents at the back-propagation sites before induction. The same data set as that shown in Table 1 was used. The line represents the best linear fit of the data (correlation coefficient, $r=0.87, P<0.001$, and $r=-0.36, P=0.16$ in $\mathbf{a}$ and $\mathbf{b}$, respectively). Red symbols represent serial triplets that did not contain the reciprocal connection $E_{2-1}$ (Fig. 3). c. The degree of depression for the induced LTD (black) or forward-propagated depression (red) is plotted against the initial amplitude of the synaptic current at the site of induction or the forward-propagation site, respectively. Triangles and circles represent glutamatergic and GABAergic synapses, respectively. Lines represent the best linear fits to the data (black, $r=-0.02, P=0.9$; red, $r=0.32, P=0.3$ ). $\mathbf{d}$, The degree of depression at the back-propagated site is plotted against that observed at the site of LTD induction for each triplet. Triangles and circles represent glutamatergic and GABAergic synapses, respectively. Lines represent best linear fits to the data $(r=0.20$, $P=0.5)$. e, The degree of depression at the presynaptic propagated sites and LTD induction sites was plotted for each triplet network examined for glutamatergic synapses. The line represents the best fit to the data $(r=0.80, P=0.005)$. $\mathbf{f}$, The degree of depression at the propagation and induction sites was plotted for postsynaptic lateral propagation of LTD to glutamatergic (triangles) and GABAergic (circles) synapses. Red and black symbols represent data for which LTD was induced at a glutamatergic or GABAergic synapse, respectively, and the corresponding lines represent the best linear fits to the data (red, $r=0.13, P=0.7$; black, $r=-0.28, P=0.6)$. 
that the nature of cellular signals underlying various forms of preand postsynaptic propagation of depression is likely to be different.

\section{Information flow in a neural network}

Information coded by changes in the membrane potential flows rapidly from dendrites to the axon and across the synapse to the postsynaptic neuron in an anterograde direction. Retrograde signals also flow across the synapse on a slower timescale to regulate the development, maintenance and modification of the properties of the presynaptic neuron ${ }^{1,37-41}$. Gradual loss of synapses at the dendrites after axotomy ${ }^{42-44}$ or interruption of axonal transport ${ }^{45}$ has demonstrated the existence of slow axon-dendritic trophic interaction of the order of days to weeks which is crucial for maintaining the structural and functional integrity of synaptic inputs at the dendrites. Our results on the back propagation of synaptic depression further indicate an axon-dendritic signalling that conveys information on activity-dependent synaptic modification at the axonal terminals with a timescale of the order of minutes. In addition to their role in integrating synaptic potentials, dendrites may also integrate retrograde signals on the efficacy of the axonal outputs of a neuron. Further investigation is necessary to determine whether synaptic potentiation also undergoes back propagation, and whether back-propagated influences from multiple axonal outputs of the neuron can be integrated at its dendrites.

The extensive propagation of synaptic modification in glutamatergic neurons suggests that local excitatory activity within the neural network may result in a more extensive distribution of longterm synaptic modification in the network than that induced by repetitive activities at GABAergic synapses. Lateral propagation of depression, either pre- or postsynaptic, will result in a loss of pathway specificity associated with LTD. This loss of specificity appears to contradict findings on LTD induced at CA1 regions of hippocampal slices, where only synapses made by the activated input were depressed, but non-activated inputs remained unaffected $^{7,28,29}$. However, these latter experiments were usually done using well-separated inputs to the CA1 region. Lateral propagation of LTD may be spatially restricted, in a way similar to that found for the lateral spread of LTP in hippocampal slice $e^{9,10}$ and slice cultures ${ }^{8,11}$. The extensive propagation of depression observed here may reflect the restricted geometry of nerve processes and higher density of connectivity between neurons in an artificial culture condition. Although the phenomena in cell culture may be an exaggeration of those occuring in vivo, our simple neural networks will enable investigation of network properties that may be present to varying degrees in more complex neural systems.

Artificial neural networks with multiple layers of interconnected 'neuronal' units and modifiable synaptic connections have been used for modelling learning processes of the nervous system ${ }^{23}$. Such a network can be trained by reiterative adjustments of the weight of synaptic connections between units of different layers to reduce the errors in the output. An efficient computational procedure for supervised learning in simple feedforward networks, known as the back-propagation algorithm ${ }^{22,46}$, uses the error in the output to compute adjustments to the weights of synaptic connections from upstream units within the network. The biological plausibility of such an algorithm requires information flow from output synapses of a neuron back towards its input synapses. Although more complex feedback circuits may be used to convey information in a retrograde direction ${ }^{47}$, the back propagation of synaptic modification demonstrated here offers an efficient cellular mechanism for the implementation of a powerful learning algorithm in a simple biological neural network.

\section{Methods}

Cell culture. Low-density cultures of dissociated embryonic rat hippocampal neurons were prepared as described ${ }^{24}$. Briefly, hippocampi were removed from E18-20 embryonic rats and treated with trypsin for $20 \mathrm{~min}$ at $37^{\circ} \mathrm{C}$, followed by washing and gentle trituration. The dissociated cells were plated at densities of 30,000 to 50,000 cells ml $\mathrm{m}^{-1}$ on poly-L-lysine-coated glass coverslips in $35-\mathrm{mm}$ Petri dishes. The plating medium was Dulbecco's minimum essential medium (DMEM; BioWhittaker) supplemented with 10\% calf serum (Hyclone), $10 \%$ Ham's F12 with glutamine (BioWhittaker) and $50 \mathrm{U} \mathrm{ml}^{-1}$ penicillin-streptomycin (Sigma). The culture medium was changed to the above medium containing $20 \mathrm{mM} \mathrm{KCl} 24 \mathrm{~h}$ after plating. Both glial and neuronal cell types are present under these culture conditions. Cells were used for electrophysiological recordings after 10-14 days in culture.

Electrophysiology. Whole-cell perforated patch recordings $s^{48-50}$ from three hippocampal neurons were performed simultaneously, using amphotericin B (Sigma) for perforation. The micropipettes were made from borosilicate glass capillaries (Kimax), with a resistance in the range of 2-4 M 2 . The pipettes were tip-filled with internal solution and then back-filled with internal solution containing $150 \mathrm{ng} \mathrm{ml}^{-1}$ amphotericin B. The internal solution contained the following (in mM): potassium gluconate 136.5; $\mathrm{KCl} 17.5 ; \mathrm{NaCl} 9 ; \mathrm{MgCl}_{2} 1$; HEPES 10; EGTA 0.2 ( $\mathrm{pH} 7.30)$. The external bath solution was a HEPESbuffered saline (HBS) containing the following (in $\mathrm{mM}$ ): $\mathrm{NaCl} \mathrm{145;} \mathrm{KCl} 3$; HEPES 10; $\mathrm{CaCl}_{2} 3$; glucose $8 ; \mathrm{MgCl}_{2} 2$ (pH 7.30). The bath was constantly perfused with fresh recording medium at a slow rate throughout the recording; all experiments were done at room temperature. Neurons were visualized by phase-contrast microscopy with a Nikon inverted microscope. Recordings were made with three patch-clamp amplifiers (Axopatch 200; Axon Instruments). Signals were filtered at $5 \mathrm{kHz}$ using amplifier circuitry and stored on a VCR. Data were sampled at $10 \mathrm{kHz}$ and analysed using a pClamp 6.0 software (Axon Instruments). Series resistance $(10-30 \mathrm{M} \Omega$ ) was always compensated at 75$80 \%$ (lag, $10 \mu \mathrm{s}$ ). Data from experiments were accepted for analysis only in cases where the postsynaptic currents did not vary beyond $10 \%$ of the average values during the control period and the input resistance (100-300 M $\Omega$ ) remained constant throughout the experiment. For assaying synaptic connectivity, each neuron was stimulated at a low frequency $(0.06 \mathrm{~Hz})$ by $1-\mathrm{ms}$ step depolarization from $-80 \mathrm{mV}$ to $+20 \mathrm{mV}$ in voltage-clamp mode, and the responses from the other two neurons as well as autaptic responses in the stimulated neuron itself were recorded. The use of potassium gluconate as the principal ionic constituent in the intracellular solution resulted in inward EPSCs and IPSCs at resting membrane potentials $(-80$ to $-60 \mathrm{mV})$. The IPSCs have distinctly longer decay times and more negative reversal potentials than EPSCs. Autaptic currents, when present, followed the 1-ms step depolarization in voltage-clamp mode. Consistent with previous reports ${ }^{24,26}$, EPSCs and IPSCs in these cultures are glutamatergic and GABAergic in nature, respectively. As shown in Fig. 2a, b, pharmacological studies indicated that EPSCs are mediated by AMPA and NMDA receptors, blocked by receptor antagonists (土)-2amino-5-phosphonopentanoic acid (AP5, $50 \mu \mathrm{M}$; Research Biochemicals International and 6-cyano-7-nitroquinoxaline-2,3-dione (CNQX, $10 \mu \mathrm{M}$, Research Biochemicals International); IPSCs are mediated by $\mathrm{GABA}_{\mathrm{A}}$ receptors, blocked by bicuculline methiodide $(10 \mu \mathrm{M}$, Research Biochemicals International). For all triplets consisting of divergent outputs (configuration 2 in Table 1), in no case did we observe a single neuron making both glutamatergic and GABAergic synapses with other neurons, consistent with the expected uniformity in the transmitter type used at all axonal terminals of the same neuron.

Received 31 December 1996; accepted 11 June 1997.

1. Purves, D. \& Lichtman, J. W. Principles of Neural Development (Sinauer Associates, Sunderland, MA, 1985).

. Goodman, C. \& Shatz, C. J. Developmental mechanisms that generate precise patterns of neuronal connectivity. Cell/Neuron 72/10, 77-98 (1993).

3. Katz, L. C. \& Shatz, C. J. Synaptic activity and the construction of cortical circuits. Science 274, $1133-$ 1138 (1996).

Bliss, T. V. \& Lømo, T. Long-lasting potentiation of synaptic transmission in the dentate area of the anaesthetized rabbit following stimulation of the perforant path. J. Physiol. (Lond.) 232, 331-356 (1973)

5. Ito, M. The cellular basis of cerebellar plasticity. Curr. Opin. Neurobiol. 1, 616-620 (1991)

6. Bliss, T. V. \& Collingridge, G. L. A synaptic model of memory: long-term potentiation in the hippocampus. Nature 361, 31-39 (1993).

7. Linden, D. J. \& Connor, J. A. Long-term synaptic depression. Annu. Rev. Neurosci. 18, 319-357 (1995).

8. Bonhoeffer, T., Staiger, V. \& Aertsen, A. Synaptic plasticity in rat hippocampal slice cultures: local "Hebbian" conjugation of pre- and postsynaptic stimulation leads to distributed synaptic enhancement. Proc. Natl Acad. Sci. USA 86, 8113-8117 (1989).

9. Schuman, E. M. \& Madison, D. V. Locally distributed synaptic potentiation in the hippocampus. Science 263, 532-536 (1994).

10. Kossel, A., Bonhoeffer, T. \& Bolz, J. Non-Hebbian synapses in rat visual cortex. Neuroreport 1, 115118 (1990). 
11. Engert, F. \& Bonhoeffer, T. Synapse specificity of long-term potentiation breaks down at shor distances. Nature 388, 279-284 (1997).

12. Scanziani, M., Malenka, R. C. \& Nicoll, R. A. Role of intercellular interactions in heterosynaptic longterm depression. Nature 380, 446-450 (1996).

13. McMahon, L. L. \& Kauer, J. A. Hippocampal interneurons express a novel form of synaptic plasticity. Neuron 18, 266, 737-739 (1977).

14. Lynch, G. S., Dunwiddle, T. \& Gribkoff, V. Heterosynaptic depression: a postsynaptic correlate of long-term potentiation. Nature 266, 737-739 (1977).

15. Abraham, W. C. \& Goddard, G. V. Asymmetric relationships between homosynaptic long-term potentiation and heterosynaptic long-term depression. Nature 305, 717-719 (1983).

16. Christie, B. R. \& Abraham, W. C. NMDA-dependent heterosynaptic long-term depression in the dentate gyrus of anaesthetized rats. Synapse 10, 1-6 (1992).

17. Otani, S., Connor, J. A. \& Levy, W. B. Long-term potentiation and evidence for novel synaptic association in CAl stratum-oriens of rat hippocampus. Learn. Mem. 2, 101-106 (1995).

18. Muller, D., Hefft, S. \& Figurov, A. Heterosynaptic interactions between LTP and LTD in cal hippocampal slices. Neuron 14, 599-605 (1995).

19. Lo, Y. \& Poo, M.-m. Activity-dependent synaptic competition in vitro: Heterosynaptic suppression of developing synapses. Science 254, 1019-1022 (1991).

20. Cash, S., Zucker, R. S. \& Poo, M.-m. Spread of synaptic depression by presynaptic cytosolic signaling. Science 272, 998-1001 (1996).

21. Vincent, P. \& Marty, A. Neighboring cerebellar Purkinje cells communicate via retrograde inhibition of common presynaptic interneurons. Neuron 11, 885-893 (1993).

22. Rumelhard, D. E., Hinton, G. E. \& Williams, R. J. Parallel Distributed Processing: Explorations in the Microstructure of Cognition Vol. 1 (eds Rumelhart, D. E. \& McClelland, J. L.) 316-362 (MIT Press, Cambridge, MA, 1986).

23. Churchland, P. S. \& Sejnowski, T. J. The Computational Brain (MIT Press, Cambridge, MA, 1992).

24. Wilcox, K. S., Buchhalter, J. \& Dichter, M. A. Properties of inhibitory and excitatory synapses between hippocampal neurons in very low density cultures. Synapse 18, 128-151 (1994).

25. Bekkers, J. M. \& Stevens, C. F. Excitatory and inhibitory autaptic currents in isolated hippocampal neurons maintained in cell culture. Proc. Natl Acad. Sci. USA 88, 7834-7838 (1991).

26. Goda, Y. \& Stevens, C. F. Long-term depression properties in a simple system. Neuron 16, 103-111 (1996)

27. Tong, G., Malenka, R. C. \& Nicoll, R. A. Long-term potentiation in cultures of single hippocampal granule cells: a presynaptic form of plasticity. Neuron 16, 1147-1157 (1996)

28. Dudek, S. M. \& Bear, M. F. Homosynaptic long-term depresison in area CAl of hippocampus and effects of $N$-methyl-D-aspartate receptor blockade. Proc. Natl Acad. Sci. USA 89, 4363-4367 (1992)

29. Mulkey, R. M. \& Malenka, R. C. Mechanisms underlying induction of homosynaptic long-term depression in area CAl of the hippocampus. Neuron 9, 967-975 (1992).

30. Bolshakov, V. Y. \& Siegelbaum, S. A. Postsynaptic induction and presynaptic expression of hippocampal long-term depression. Science 264, 1148-1751 (1994).

31. Stevens, C. F. \& Wang, Y. Changes in reliabiity of synaptic function as a mechanism for plasticity. Nature 371, 704-707 (1994)

32. Xiao, M.-Y., Wigstrom, H. \& Gustafsson, B. Long-term depression in the hippocampal CA1 region is associated with equal changes in AMPA and NMDA receptor-mediated synaptic potentials. Eur. J. Neurosci. 6, 1055-1057 (1994).
33. Dan, Y. \& Poo, M.-m. Hebbian depression of isolated neuromuscular synapses in vitro. Science 256, $1570-1573$ (1992).

34. Cash, S., Dan, Y., Poo, M.-m. \& Zucker, R. Depression of developing neuromuscular synapses by postsynaptic elevation of calcium. Neuron 16, 745-754 (1996).

35. Brocher, S., Artola, A. \& Singer, W. Intracellular injection of $\mathrm{Ca}^{2+}$ chelators blocks induction of longterm depression in rat visual cortex. Proc. Natl Acad. Sci. USA 89, 123-127 (1992).

36. Murthy, V. N., Sejnowski, T. J., Stevens, C. F. Heterogenous release properties of visualized individual hippocampal synapses. Neuron 18, 599-612 (1997).

37. Clarke, P. G. H. The role of trophic communication in biological networks. Concepts Neurosci. 2, 201219 (1991).

38. Jessell, T. M. \& Kandel, E. R. Synaptic transmission: a bidirectional and self-modifiable form of cellcell communicaiton. Cell/Neuron 72/10, 1-30 (1993).

39. Dan, Y. \& Poo, M.-m. Retrograde interactions during formation and elimination of neuromuscular synapses. Curr. Opin. Neurobiol. 4, 95-100 (1994).

40. Davis, G. W. \& Murphey, R. K. Retrograde signaling and the development of transmitter release properties in the invertebrate nervous system. J. Neurobiol. 25, 740-756 (1994).

41. Fitzsimonds, R. M. \& Poo, M.-m. Retrograde signaling in the development and modulation of synapses. Physiol. Rev. (in the press).

42. Mathews, M. R. \& Nelson, V. H. Detachment of structurally intact nerve endings from chromatolytic neurons of rat superior cervical ganglion during the depression of synaptic transmission induced by post-ganglionic axotomy. J. Physiol. (Lond.) 245, 91-135 (1975)

43. Purves, D. Functional and structural changes in mammalian sympathetic neurones following interruption of their axons. J. Physiol. (Lond.) 252, 429-463 (1975).

44. Wood, M. R. \& Faber, D. S. Electrohysiological and morphological correlates of axotomy-induced deafferentation of the goldfish Mauthner cell. J. Comp. Neurol. 244, 413-426 (1986).

45. Pilar, G. \& Landmesser, L. Axotomy mimicked by localized colchicine application. Science 177, 11161118 (1972).

46. Rumelhart, D. E., Hinton, G. E. \& Williams, R. J. Learning representations by back-propagation errors. Nature 323, 533-536 (1986).

47. Zipser, D. \& Rumelhart, D. E. in Computational Neuroscience (ed. Schwartz, E. L.) 192-200 (MIT Press, Cambridge, MA, 1990).

48. Hamill, O. P., Marty, A., Neher, E., Sakmann, B. \& Sigworth, F. J. Improved patch-clamp techniques for high-resolution current recording from cells and cell-free membrane patches. Pflügers Arch. 391, 85-100 (1981).

49. Horn, R. \& Marty, A. Muscarinic activation of ionic currents measured by a new whole-cell recording method. J. Gen. Physiol. 92, 145-159 (1988).

50. Rae, J., Cooper, K., Gates, P. \& Watsky, M. Low access resistance perforated patch recordings using amphotericin B. J. Neurosci. Meth. 37, 15-26 (1991).

Acknowledgements. We thank T. Mau for preparing cell cultures, and Y. Dan, B. Berninger and A. Schinder for discussion. This work was supported by grants from the NIH and the NSF.

Correspondence and requests for materials should be addressed to M.-m.P. (e-mail: mpoo@ucsd.edu).

\section{YOURS TO HAVE AND TI \\ The publication you are reading is protected by copyright law. \\ Photocopying copyright material \\ If you take photocopies from books, magazines and periodicals at work your employer should be licensed

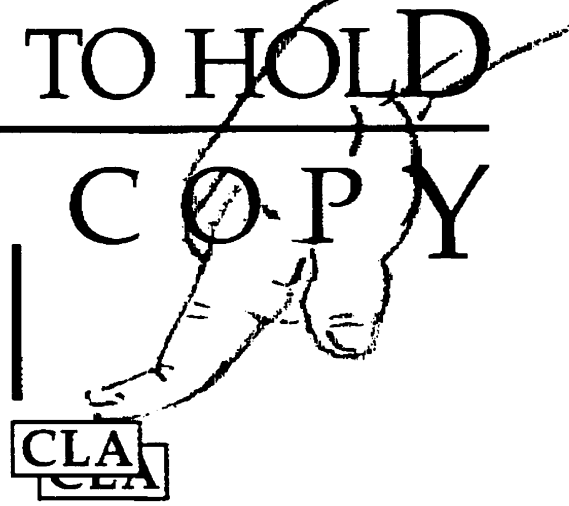
without permission is no different from stealing a magazine from a newsagent, only it doesn't seem like theft. with CLA.

Make sure you are protected by a photocopying licence.
The Copyright Licensing Agency Limited 90 Tottenham Court Road, London WIP OLP Telephone: 01714365931 Fax: 01714363986 